\title{
Prediction of left ventricular systolic dysfunction after 6 months of aortic valve replacement in patients with chronic severe aortic regurge
}

\begin{abstract}
Introduction: LVEF as a surrogate for myocardial performance is problematic in chronic $\mathrm{AR}$ as it is augmented by increased preload. Therefore, we need noninvasive parameters to assess myocardial function in altered loading conditions. We aim to assess the validity of new echo-Doppler indices for prediction of postoperative LV dysfunction 6 months after AVR.

Methods: We recruited 20 patients with severe isolated AR with $\mathrm{LVEF}>50 \%$, prepared for AVR. Echocardiographic examination was done 48hours before and 6 months after AVR. Early diastolic driving force "DF", Global longitudinal strain "GLS" and Left ventricular ejection fraction "LVEF" by modified biplane Simpson method were measured. Our patients were classified according to postoperative LVEF into Group A (15 cases); normal (postoperative $\mathrm{LVEF} \geq 50 \%$ ) and Group B (5 cases); postoperative LV systolic dysfunction (postoperative $\mathrm{LVEF}<50 \%$ ).

Results: preoperative DF was $0.20 \pm 0.12$ Newton in group A while it was $0.66 \pm 0.28$ Newton in group $\mathrm{B}$, this difference was statistically significant $(\mathrm{t}=3.5, \mathrm{p}<0.05)$. EF was $62.73 \pm 6.64 \%$ in-group A while it was $53.2 \pm 2.77 \%$ in-group $B$, this difference was statistically highly significant $(\mathrm{t}=4.50, \mathrm{p}<0.001)$. GLS was $-18.95 \pm 2.55 \%$ in-group A while it was $-12.56 \pm 2.04 \%$ in group $\mathrm{B}$, this difference was statistically highly significant $(\mathrm{t}=5.04$, $\mathrm{p}<0.001$ ). On plotting the ROC curves, it was clear that preoperative GLS and DF are strong predictors of post-operative systolic dysfunction in such cases.
\end{abstract}

Conclusion: Preoperative GLS and DF seem to be independent predictors for postoperative LV systolic dysfunction after AVR for chronic severe AR.

Keywords: aortic regurgitation, aortic valve replacement, color doppler, diastolic function
Volume 12 Issue 5 - 2019

\author{
Mohammad Gouda,Ahmed S Ammar \\ Mohammad Hassan, MF Abdel-Alwahab \\ Cardiology Department, Faculty of Medicine, Zagazig University, \\ Egypt \\ Correspondence: Dr. Mohammad Gouda, MD. Assistant \\ Professor of Cardiology, Postal Address: Egypt, Zagazig, Zagazig \\ University, Faculty of Medicine, Cardiology Department, 44519 , \\ Tel +21019693393, Fax +20552357770, \\ Email cardioman77@yahoo.com
}

Received: August 16,2019 | Published: September 21, 2019
Abbreviations: AVR, severe aortic valve regurgitation; LV, left ventricular; LVFR, Left ventricular ejection fraction; GLS, global longitudinal strain; DT, deceleration time; ROC, receiver operating characteristic; DF, driving force; LRA, logistic regression analysis

\section{Introduction}

The natural history of chronic severe aortic valve regurgitation (AR) is characterized by a prolonged period of preserved global left ventricular (LV) systolic function and compensated eccentric hypertrophy, which insidiously gives way to myocardial decompensation. ${ }^{1}$ In its early stages, myocardial dysfunction may be reversible once implantation of a mechanical or bioprosthetic aortic valve eliminates the volume load. If left untreated, myocardial dysfunction becomes irreversible, a condition associated with high rates of morbidity and mortality. ${ }^{2}$ The choice of optimal timing of aortic valve replacement (AVR) in patients with AR often poses a dilemma for clinicians. If AVR is performed at a time when LV function is normal, then the patient may be exposed unnecessarily to the potential hazards that accompany prosthetic valves. However, if surgical correction of the volume overload state is delayed excessively irreversible cardiac dysfunction may have already occurred and the outcome may be unsatisfactory. ${ }^{3}$ The decision is to when to intervene for chronic AR depends primarily on the symptomatic status of the patient and the potential for normalization of ventricular and myocardial performance after AVR. ${ }^{4}$ Measuring LVEF as a surrogate for myocardial performance is problematic in the setting of chronic AR because LVEF is augmented by increased preload and is therefore limited in detecting subtle myocardial dysfunction. Thus, there is a clinical need for quantitative noninvasive parameters to assess myocardial function in the setting of altered loading conditions and to predict postoperative LV systolic dysfunction. ${ }^{5}$ The aim of the study is to assess the validity of new echo-Doppler indices for prediction of postoperative LV dysfunction 6 months after AVR.

\section{Methods}

This study had been carried out in Cardiology Department, Zagazig University. It included 20 patients with severe isolated aortic regurgitation with LVEF $>50 \%$, prepared for AVR. We excluded patients with other significant valve disease (more than mild degree), associated congenital heart disease, and coronary artery disease with a significant stenosis, previous cardiac surgery and atrial fibrillation. Transthoracic echocardiographic examination was done before and 6 months after AVR. The following measures were selected for analysis:

\section{a. Assessment of AR severity}

Vena contarcta width, JET width, AR pressure half time. (Severe AR was diagnosed when vena contracta $>0.6 \mathrm{~cm}$, JET width $>65 \%$ of LVOT, PHT $<200$ milliseconds). ${ }^{6}$ 


\section{b. Echocardiographic $L V$ function indices}

Performed $48 \mathrm{~h}$ before \& 6 months after AVR.

\section{c. The early diastolic driving force}

The early diastolic driving force calculated from the equation: $\mathrm{DF}=0.004 \mathrm{E}^{2} / \mathrm{DT}$ Newton. ${ }^{7} \mathrm{E}$ wave (early diastolic mitral inflow velocity), DT (deceleration time) measured by pulsed wave Doppler imaging at mitral leaflet tips in 4-chamber view as the time-interval between peak of $\mathrm{E}$ wave and the point where the descending limb touches baseline.

\section{d. Global longitudinal strain (GLS}

Global longitudinal strain by speckle tracking; GLS evaluates the shortening and lengthening of the myocardial wall, measured from the three apical views (long axis, two and four chamber views): GLS was calculated by averaging the peak strain values of 17 segments. ${ }^{8}$ Normal regional peak systolic strain in the longitudinal direction is approximately $-18 \%{ }^{9}$

\section{e. $\mathrm{E} / \mathrm{è}$}

Ratio of early diastolic mitral inflow velocity (measured by pulsed wave Doppler imaging with sample volume set at the mitral leaflet tips in four chamber view) to early diastolic mitral annular velocity (measured by pulsed wave tissue Doppler imaging with sample volume set at the septal, lateral mitral annulus in four chamber view).

\section{f. Left ventricular ejection fraction (LVEF)}

Left ventricular ejection fraction by modified biplane method of Simpson. 2 orthogonal views - apical four-chamber and apical twochamber - and manual tracing of endocardial borders manually traced at end systole and end-diastole are needed. ${ }^{10}$ It is calculated from the formula: $L V E F=(L V E D V-L V E S V) \times 100 / L V E D V$. Normally it is 50$70 \%$, LV systolic dysfunction is said if $\mathrm{LVEF}<50 \%$. ${ }^{11}$

The patients were classified according to postoperative $L V$ systolic function (postoperative LVEF) into:

i. Group A (15 cases): Normal postoperative LV systolic function (postoperative LVEF $\geq 50 \%$ )

ii. Group B (5 cases): Postoperative LV systolic dysfunction (postoperative LVEF $<50 \%$ )

\section{Statistical analysis}

Statistical presentation and analysis of the present study was conducted, using the mean, standard deviation, paired $(\mathrm{T})$ test, independent $t$ test, and chi square by SPSS V 20. Regression analysis was performed to determine the independent predictors of postoperative LV systolic dysfunction. Receiver operating characteristic (ROC) curve analysis was performed to determine cutoff values that predict postoperative LV systolic dysfunction. $\mathrm{P}$ value $<0.05$ was considered as statistically significant.

\section{Results}

Demographically; we had 11 female patients (55\%) and 9 male patients (45\%). In group A, we had 8 females and 7 males while in group $\mathrm{B}$ we had 3 females and 2 males, there was no significant difference concerning the gender between both groups $(X=0.09, p=0.77)$. The overall age of our study cases ranged from 19-45years with mean $32.55 \pm 7.8$ years. The age of cases in-group A was $29.80 \pm 6.74$ years while in-group B it was $40.8 \pm 4.02$ years, that represents a statistically significant difference $(\mathrm{t}=-3.41, \mathrm{p}=0.003)$ Table 1 .

Table I Differentiation of patients according to demographic characteristics in relation to study groups

\begin{tabular}{|c|c|c|c|c|c|c|}
\hline \multirow{2}{*}{ Characteristics } & \multicolumn{4}{|c|}{ Age } & \multirow{2}{*}{$(\mathrm{t})$} & \multirow{2}{*}{$\mathbf{P}$} \\
\hline & \multicolumn{4}{|c|}{ Mean $\pm S D$} & & \\
\hline Group A $(n=15)$ & \multicolumn{4}{|c|}{$29.80 \pm 6.74$} & \multirow{2}{*}{-3.41} & \multirow{2}{*}{$0.003^{*}$} \\
\hline Group B $(n=5)$ & \multicolumn{4}{|c|}{$40.8 \pm 4.02$} & & \\
\hline \multirow{2}{*}{ Characteristics } & \multicolumn{2}{|c|}{ Female } & \multicolumn{2}{|c|}{ Male } & \multirow{2}{*}{$\chi^{2}$} & \multirow{2}{*}{$\mathrm{P}$} \\
\hline & No & $\%$ & No & $\%$ & & \\
\hline \multirow[t]{2}{*}{ Group A $(n=15)$} & 8 & 53.3 & 7 & 46.7 & & \\
\hline & & & & & & 0.77 \\
\hline Group B $(n=5)$ & 3 & 60 & 2 & 40 & 0.09 & NS \\
\hline
\end{tabular}

Etiologically; $55 \%$ of our cases were rheumatic, $25 \%$ had Bicuspid AV and finally $20 \%$ of them had aneurysm of ascending aorta. There was no statistical significant differences between cases in both groups $(\mathrm{X}=0.09, \mathrm{p}>0.05)$.

Concerning the preoperative measured Echo-Doppler indices, the driving force (DF) was $0.20 \pm 0.12$ Newton in group A while it was $0.66 \pm 0.28$ Newton in group B this difference was statistically significant $(\mathrm{t}=3.5, \mathrm{p}<0.05)$. Regarding $\mathrm{E} / \mathrm{e}$, it was $11.95 \pm 5.52$ ingroup $\mathrm{A}$ while it was $21.12 \pm 3.43 \mathrm{in}$-group $\mathrm{B}$, this difference was statistically highly significant $(\mathrm{t}=4.38, \mathrm{p}<0.001)$. Concerning the $\mathrm{EF}$, it was $62.73 \pm 6.64 \%$ in-group A while it was $53.2 \pm 2.77 \%$ ingroup $\mathrm{B}$, this difference was statistically highly significant $(\mathrm{t}=4.50$, $\mathrm{p}<0.001)$. Lastly, $G L S$, it was $-18.95 \pm 2.55 \%$ in group A while it was $-12.56 \pm 2.04 \%$ in group $\mathrm{B}$, this difference was statistically highly significant $(\mathrm{t}=5.04, \mathrm{p}<0.001)$ Table 2 .

Table 2 Comparative study of preoperative echocardiographic indices between both groups

\begin{tabular}{lllll}
\hline Variable & $\begin{array}{l}\text { Group A } \\
(\mathbf{n}=15)\end{array}$ & $\begin{array}{l}\text { Group B } \\
(\mathbf{n}=5)\end{array}$ & Test & $\mathbf{P}$ \\
\hline $\begin{array}{l}\text { Driving force } \\
\text { E/è }\end{array}$ & $0.20 \pm 0.12$ & $0.66 \pm 0.28$ & 3.5 & $<0.05^{*}$ \\
$\begin{array}{l}\text { Ejection } \\
\text { fraction }\end{array}$ & $11.95 \pm 5.52$ & $21.12 \pm 3.43$ & 4.38 & $<0.00 I^{* *}$ \\
$\begin{array}{l}\text { Global } \\
\text { longitudinal } \\
\text { strain }\end{array}$ & $-18.95 \pm 6.64$ & $53.2 \pm 2.77$ & 4.5 & $<0.00 I^{* *}$ \\
& 2.55 & $-12.56 \pm$ & & \\
\hline
\end{tabular}

Correlation studies: There was a highly significant negative correlation between post-operative Ejection fraction and preoperative GLS $(\mathrm{R}=-0.901, \mathrm{P}<0.001)$, preoperative $\mathrm{DF}(\mathrm{r}=-0.852$, $\mathrm{p}<0.001)$ and pre-operative $\mathrm{E} / \mathrm{e}(\mathrm{r}=-0.806, \mathrm{p}<0.001)$. In addition, there was significant negative correlation between post-operative Ejection fraction and ageing $(\mathrm{r}=-0.601, \mathrm{p}<0.05)$ while there was a significant positive correlation between post-operative Ejection fraction preoperative Ejection fraction $(\mathrm{r}=0.641, \mathrm{p}<0.05)$ Table 3, Figure 1 . 
Table 3 Correlation of post-operative LVEF with other parameters

\begin{tabular}{llc}
\hline \multirow{2}{*}{ Pre-operative indices } & \multicolumn{2}{l}{ Postoperative LVEF } \\
\cline { 2 - 3 } & $\mathrm{r}$ & $\mathrm{P}$ \\
\hline DF & -0.852 & $<0.00 I^{* *}$ \\
E/è & -0.806 & $<0.00 I^{* *}$ \\
EF & $0.64 \mathrm{I}$ & $<0.05^{*}$ \\
GLS & -0.901 & $<0.00 I^{* *}$ \\
Age & -0.601 & $<0.05^{*}$ \\
\hline
\end{tabular}

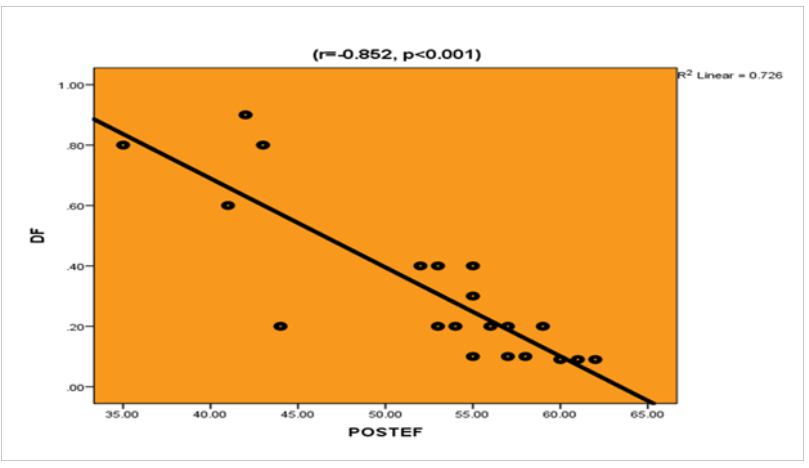

Figure I Correlation of post-operative left ventricular ejection fraction and pre-operative driving force.

On plotting the ROC curves, it was clear that preoperative GLS (AUC $=99 \%, p<0.001$, cut off value $=-14 \%$ ), preoperative DF (AUC $=89 \%, \mathrm{p}<0.05$, cut off value $=0.5$ newton) and preoperative $\mathrm{EF}$ (AUC $=87 \%, \mathrm{p}<0.05$, cut off value $=55 \%$ ) are strong predictors that can predict the occurrence of post-operative systolic dysfunction in such cases Table 4, Figures $2 \& 3$.

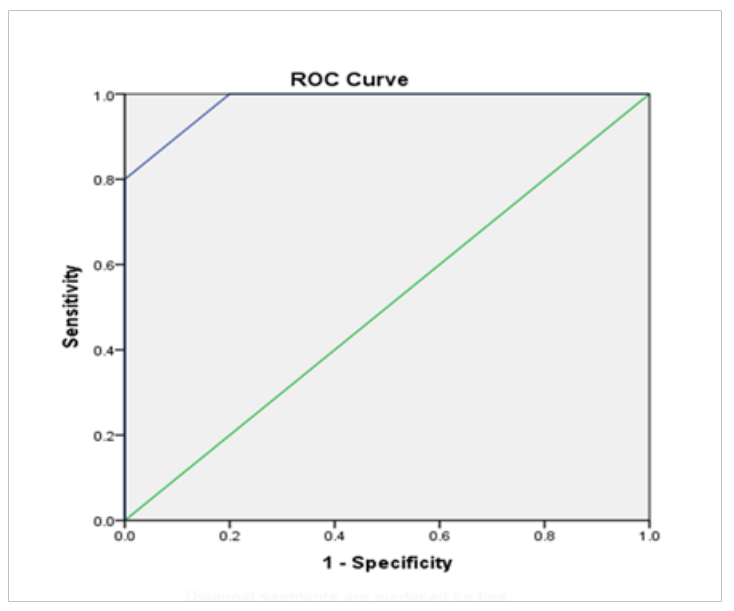

Figure 2 ROC curve of preoperative GLS as a predictor of post-operative systolic dysfunction.

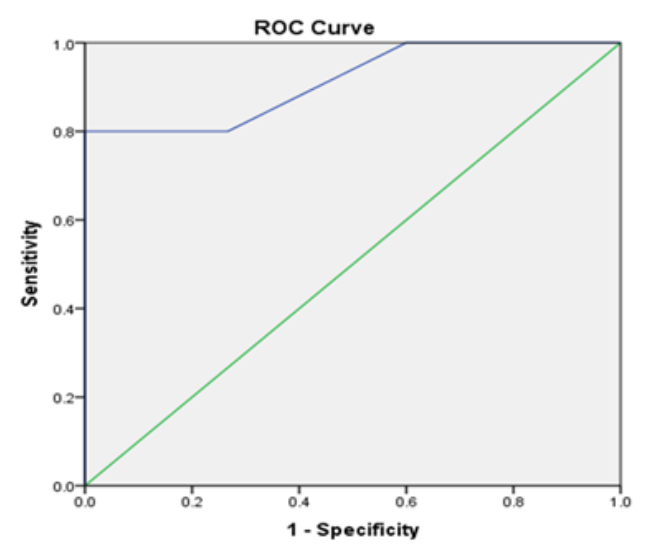

Figure 3 ROC curve of preoperative Driving force (DF) as a predictor of post-operative systolic dysfunction.

Table 4 Reliability data of different preoperative Echo Doppler indices as predictors for postoperative Systolic dysfunction

\begin{tabular}{|c|c|c|c|c|c|c|c|}
\hline & Cut off & AUC & $\mathbf{p}$ & $95 \% \mathrm{Cl}$ & Sensitivity & Specificity & Accuracy \\
\hline DF & 0.5 & 0.89 & $<0.05^{*}$ & $0.69-1.09$ & 80 & 100 & 95 \\
\hline E/è & 18 & 0.67 & $>0.05$ & $0.36-0.96$ & 80 & 59.7 & 60 \\
\hline EF & 55 & 0.87 & $<0.05^{*}$ & $0.72-1.03$ & 80 & 73.3 & 75 \\
\hline GLS & -14 & 0.99 & $<0.001^{* *}$ & $0.95-1.02$ & 80 & 100 & 95 \\
\hline
\end{tabular}

DF, Driving force; EF, Ejection fraction; GLS, Global longitudinal strain

Citation: Gouda M, Hassan ASAM, Abdel-Alwahab MF. Prediction of left ventricular systolic dysfunction after 6 months of aortic valve replacement in patients with chronic severe aortic regurge. / Cardiol Curr Res. 2019;12(5): I I2-I I6. DOI: 10.15406/jccr.2019.12.0045 I 
In order to detect the strongest predictor of post-operative systolic dysfunction in our cases, we performed the logistic regression analysis (LRA). It was clear that preoperative GLS was the strongest predictor of post-operative systolic dysfunction $(\mathrm{R}=-0.09, \mathrm{p}<0.001)$ followed by preoperative $\mathrm{DF}(\mathrm{R}=0.98, \mathrm{p}<0.05)$ Table 5 .

Table 5 Logistic regression Analysis of significant variables (different preoperative Echo Doppler indices) for postoperative systolic dysfunction

\begin{tabular}{llll}
\hline Variables & Regression Coefficient & SE & $\mathbf{P}$ \\
\hline DF & 0.98 & 0.37 & $<0.05^{*}$ \\
E/è & 0.01 & 0.02 & $>0.05$ \\
EF & -0.03 & 0.01 & $<0.05^{*}$ \\
GLS & -0.09 & 0.02 & $<0.00 I^{*}$ \\
\hline
\end{tabular}

\section{Discussion}

Current guidelines indicate surgery in symptomatic patients with severe AR or, in the case of asymptomatic patients, surgery is indicated when LV function is impaired $(\mathrm{EF}<50 \%)$ and should be considered if LV end-diastolic diameter (LVEDD) is $>70 \mathrm{~mm}$ or LVESD is $>50 \mathrm{~mm}$ (or $>25 \mathrm{~mm} / \mathrm{m}^{2}$ BSA in patients with small body size). Left ventricular systolic dysfunction is initially reversible with surgery. However, AVR should be performed before onset of irreversible LV dysfunction. Despite these indications, the optimal timing of cardiac surgery for chronic AR remains challenging, because of systolic dysfunction may precedes symptom onset in more than one-fourth of patients with this condition. In addition, the increased LV end-diastolic volume and eccentric hypertrophy act as compensatory mechanisms for long periods of time, which masks the development of afterload mismatch and the progressive exhaustion of myocardial contractile reserve. ${ }^{12}$

Therefore, more sensitive parameters of early LV dysfunction are needed ${ }^{(13)}$. For detecting LV dysfunction, LV ejection fraction (LVEF) has been widely used. However, in chronic AR, because LVEF is preserved by increased preload, it cannot precisely detect the developing myocardial dysfunction ${ }^{(14)}$. A more sensitive parameter of LV dysfunction than LV diameter and LVEF would be of considerable clinical value, as it would allow clinicians to detect early abnormalities, assist in the evaluation of symptoms, and indicate the need for vigilant observation, and possibly earlier surgery ${ }^{(15)}$. Speckle-tracking strain analysis is a novel echocardiographic tool for an accurate tool to detect subclinical preoperative LV dysfunction.

Our work showed that the incidence of systolic dysfunction differs according to age, with higher incidence being in higher age groups, but it is not affected by gender. In our study, $25 \%$ of patients have developed systolic dysfunction and we found that the occurrence of postoperative LV systolic dysfunction not related to the etiology of severe AR. It has been shown that asymptomatic patients with chronic severe AR can have reduced systolic LV function while maintaining a normal global LVEF. This was explained by the fact that subendocardial fibers, which perform longitudinal LV contraction, are exposed to the increased wall stress at an early stage, and that this initiates a fibrotic process. ${ }^{16}$ This makes the strain parameters are useful to detect LV dysfunction at an earlier stage. ${ }^{17}$ This was concordant with Smedsrud et al. ${ }^{18}$ who demonstrated a reduction in GLS in 47 patients with severe AR compared with healthy control subjects $(-17.5 \pm 3.1 \% \mathrm{Vs}-22.1 \pm 1.8 \%, \mathrm{p}<0.01)$, even in the presence of a preserved LVEF. ${ }^{18}$

Using ROC analysis, we found that the optimal cutoff value of preoperative GLS for prediction of postoperative LV systolic dysfunction is $<-14 \%$ ( $80 \%$ sensitivity, $100 \%$ specificity, AUC $=99 \%)$ while Olsen et al. ${ }^{19}$ found that in medically treated patients, a GLS of $-18 \%$ was the best cutoff for the identification of disease progression while a cutoff of $-14 \%$ was predictive of poor outcome in patients undergoing AVR which agreed with our results. ${ }^{19}$

After applying regression analysis, the present study showed that preoperative Global longitudinal strain (GLS) is the most significant independent variable in prediction of postoperative LV systolic dysfunction $(p<0.001)$ also DF, EF but not $E / e^{\prime}$ are independent predictors. In this study, there is underlying systolic dysfunction which is detected by low preoperative GLS $(-17.35 \pm 3.7)$ despite normal EF (60.35 \pm 7.21$)$.

Preoperative EF has been regarded as a significant predictor for postoperative outcomes. Based on the previous reports, the EF was able to predict many adverse outcomes after surgical correction, such as the poor restoration of postoperative LV function, operative mortality ${ }^{(19)}$. In our study, we found that there is a positive correlation between preoperative LVEF \& postoperative LVEF, which is concordant with Onishi et al. ${ }^{20}$

In our study, there was a small, but significant, decrease in LVEF after AVR (60.35 \pm 7.21 vs $52.6 \pm 7.52)$. These results are concordant with those of Pomerantz et al. ${ }^{21}$ but differ from the results of others Bonow et al. ${ }^{13}$ Borer et al. ${ }^{15}$ who showed an increase in LVEF after AVR. This apparent disagreement is due to our strict inclusion of patients with normal LVEFs. Increases in LVEF in patients with AR after surgery have been shown only in patients with reduced LVEFs before surgery. ${ }^{22}$ It was showed that EF $>52 \%$ was the best cut-off value for successful early recovery of dilated LVEDD after AVR. ${ }^{23}$ This is close to our results which showed the best cutoff value of preoperative LVEF for prediction of postoperative LV systolic dysfunction (LVEF $<50 \%$ ) is $<55 \%$ ( $80 \%$ sensitivity, $73.3 \%$ specificity, accuracy $75 \%, \mathrm{AUC}=87 \%$ ). Our results showed also that preoperative LVEF was proved inferior to GLS \& DF in prediction of postoperative LV systolic dysfunction.

Up to our knowledge, No one thought to use the diastolic driving force "DF" as a predictor of outcome in patients with severe AR. In our study, DF proved to be better than E/e' to discriminate disease from normal, as with disease peak $\mathrm{E}$ changed more than peak A, but the ratio obscured the absolute differences from normal. ${ }^{24} \mathrm{DF}$ equation was later validated for prediction of end diastolic pressure. ${ }^{7}$ The present study showed that there is highly significant negative correlation between DF and postoperative LVEF. ROC analysis yielded an optimal DF cutoff value of $>0.5$ newton for prediction of postoperative LV systolic dysfunction (sensitivity $=80 \%$, specificity $100 \%$, accuracy $=95 \%, A U C=89 \%$ ). In the present work, DF has equal sensitivity $(80 \%)$ but higher specificity than EF $(100 \%$ VS $73.3 \%$ respectively), for prediction of postoperative LV systolic dysfunction. Compared to GLS, DF has equal sensitivity $(80 \%)$ and specificity $(100 \%)$ for prediction of postoperative LV systolic dysfunction. Regression analysis showed that DF is slightly inferior to GLS, but superior to EF in prediction of postoperative LV systolic dysfunction.

\section{Conclusion}

Preoperative GLS and DF seem to be independent predictors for postoperative LV systolic dysfunction after AVR for chronic severe AR. The optimal cutoff value of preoperative GLS is $<-14 \%$ ( $80 \%$ sensitivity, $100 \%$ specificity, $\mathrm{AUC}=0.99)$, while that of $\mathrm{DF}$ is $>0.5$ newton $(80 \%$ sensitivity, $100 \%$ specificity, $\mathrm{AUC}=89 \%)$. Keeping in mind the limited availability of strain software in every 
echo machine, DF is an emerging, noninvasive, simple, and reliable preoperative parameter in predicting the outcome of AVR by adjusting the timing of surgery in chronic severe AR with normal EF.

\section{Study limitations}

a. Because of the strict inclusion criteria, the number of patients was modest. So that future studies of Bigger sample size are necessary to assess our findings.

b. Surgical procedures varied widely including aortic root reimplantation, mechanical prosthesis implantation, types of prosthesis. Thus, this might have a different effect on myocardial function after surgical correction and so may influence the improvement of subclinical LV dysfunction after surgical correction.

c. Myocardial damage might occur during the surgical procedure.

d. Limited data are available about the early diastolic driving force to support our finding.

\section{Recommendations}

Wide scale use of these two echocardiography parameters in patients with chronic severe AR to discover as early as possible the ideal timing of AVR

\section{Acknowledgments}

None.

\section{Conflict of interest}

The authors declare that there is no conflict of interest.

\section{Funding}

None.

\section{References}

1. Mehmel HC, Olshausen Kv, Schuler G, et al. Estimation of left ventricular myocardial function by the ejection fraction in isolated, chronic, pure aortic regurgitation. Am J Cardiol. 1984;54(6):610-616.

2. Michel PL, Iung B, Abou Jaoude S, et al. The effect of left ventricular systolic function on long-term survival in mitral and aortic regurgitation. J Heart Valve Dis. 1995;4(suppl 2):S160-S168.

3. Stone PH, Clark RD, Goldschlager N, et al. Determinants of prognosis of patients with AR who undergo aortic valve replacement. Journal of the American College of Cardiology. 1984;3.5:1118-1126.

4. Borer JS. Aortic valve replacement for the asymptomatic patient with AR: a new piece of the strategic puzzle. Circulation. 2002;106(21):26372639 .

5. Olsen NT, Sogaard P, Larsson HB, et al. Speckle-tracking echocardiography for predicting outcome in chronic AR during conservative management and after surgery. JACC Cardiovasc Imaging. 2011;4(3):223-230

6. Tribouilloy C, Shen WF, Slama M, et al. Assessment of severity of aortic regurgitation by $\mathrm{M}$-mode colour Doppler flow imaging. Eur Heart $J$. 1991;12(3):352-356.

7. Ahmed S. Ammar, Islam A. El-sherbiny, et al. Early Diastolic Driving Force as a New Doppler Index for Prediction of Left Ventricular End Diastolic Pressure. JACC. 2014;63(12).
8. Witkowski TG, Thomas JD, Debonnaire PJ, et al. Global longitudinal strain predicts left ventricular dysfunction after mitral valve repair. Eur Heart J Cardiovasc Imaging. 2013;14(1):69-76.

9. Brown J, Jenkins C, Marwick TH. Use of myocardial strain to assess global left ventricular function: a comparison with cardiac magnetic resonance and 3-dimensional echocardiography. Am Heart $J$. 2009;157(1):102e1-102e5.

10. Otterstad JE. Measuring left ventricular volume and ejection fraction with the biplane Simpson's method. Heart. 2002;88(6):559-560.

11. Albin G, Rohko PS. Comparison of echocardiographic quantification of left ventricular ejection fraction to radio nucleotide angiography in patients with regional wall motion abnormalities. Am J Cardiol. 1990;65(15):1031-1032.

12. Joint Task Force on the Management of Valvular Heart Disease of the European Society of Cardiology (ESC), European Association for Cardio-Thoracic Surgery (EACTS), Vahanian A, et al. Guidelines on the management of valvular heart disease (version 2012). Eur Heart $J$. 2012;33(19):2451-2496

13. Bonow RO, Lakatos E, Maron BJ, et al. Serial long-term assessment of the natural history of asymptomatic patients with chronic AR and normal left ventricular systolic function. Circulation. 1991;84(4):1625-1635.

14. Corti R, Binggeli C, Turina M, et al. Predictors of long-term survival after valve replacement for chronic aortic regurgitation: is $\mathrm{M}$-mode echocardiography sufficient? Eur Heart J. 2000;22(10):866-873.

15. Borer JS, Hochreiter C, Herrold EM, et al. Prediction of indications for valve replacement among asymptomatic or minimally symptomatic patients with chronic AR and normal left ventricular performance. Circulation. 1998;97(6):525-534.

16. Paraskevaidis IA, Tsiapras D, Kyrzopoulos S, et al. The role of left ventricular long-axis contraction in patients with asymptomatic AR. $J$ Am Soc Echocardiogr. 2006;19(3):249-254.

17. Moustafa SE, Kansal M, Alharthi M, et al. Prediction of incipient left ventricular dysfunction in patients with chronic primary mitral regurgitation: a velocity vector imaging study. Eur J Echocardiogr. 2011;12(4):291-298.

18. Smedsrud MK, Pettersen E, Gjesdal O, et al. Detection of left ventricular dysfunction by global longitudinal systolic strain in patients with chronic AR. J Am Soc Echocardiogr. 2011;24(11):1253-1259.

19. Olsen NT, Sogaard P, Larsson HB, et al. Speckle-tracking echocardiography for predicting outcome in chronic AR during conservative management and after surgery. JACC Cardiovasc Imaging. 2011;4(3):223-230.

20. Onishi T, Kawai H, Tatsumi K, et al. Preoperative systolic strain rate predicts postoperative left ventricular dysfunction in patients with chronic aortic regurgitation. Circ Cardiovasc Imaging. 2010;3(2):134141.

21. Pomerantz BJ, Wollmuth JR, Krock MD, et al. Myocardial systolic strain is decreased after aortic valve replacement in patients with aortic insufficiency. Ann Thorac Surg. 2005;80(6):2186-2192.

22. Helin LM, Tamas E, Nylander E, et al. Preoperative longitudinal left ventricular function by tissue Doppler echocardiography at rest and during exercise is valuable in timing of aortic valve surgery in male AR patients. J Am Soc Echocardiogr. 2010;23(4):387-395.

23.Zhang Z, Yang J, Yu Y, et al. Preoperative ejection fraction determines early recovery of left ventricular end-diastolic dimension after aortic valve replacement for chronic severe AR. J Surg Res. 2015;196(1):4955. 